\title{
RESEARCH HIGHLGHTS: Perovskites
}

By Pabitra K. Nayak

Perovskite solar cells are at the edge of commercial success. Device efficiency records break at a regular pace, while stability and optimization are progressing rapidly. The first commercial products could reach the market within the next year, just a decade since perovskite photovoltaics were first discovered. MRS Bulletin presents coverage of the most recent impactful advances in the burgeoning field of perovskite research.

$\mathrm{H}^{\mathrm{s}}$ eterostructures with epitaxial layers, with atomically sharp interfaces, of different inorganic semiconductors such as III-V, II-VI, and transition-metal dichalcogenides are the foundation of modern electronics and optoelectronic devices. Halide perovskites, an emerging family of tunable semiconductors, have shown superb electronic and optical properties, with applications in solution-processed solar cells, light-emitting diodes (LEDs), and lasers. The easily deformable halide perovskite crystal lattice is expected to allow for a higher tolerance to lattice mismatch at semiconductor interfaces during heterostructure formation. For device miniaturization and improved performance, an atomically sharp heterostructure is essential. However, achieving atomically sharp heterostructures with halide perovskites has been a challenge. High intrinsic ion mobility causes an interdiffusion of ions, which leads to a large junction width.

In an article published in Nature (doi:10.1038/s41586-020-2219-7), Savoie, Yu, Dou, and co-workers reported overcoming the problem of interdiffusion of ions in halide perovskites by the incorporation of rigid $\pi$-conjugated organic ligands; these inhibit in-plane ion diffusion in two-dimensional (2D) halide perovskites. High-resolution transmission electron microscopy images revealed near-atomically sharp interfaces

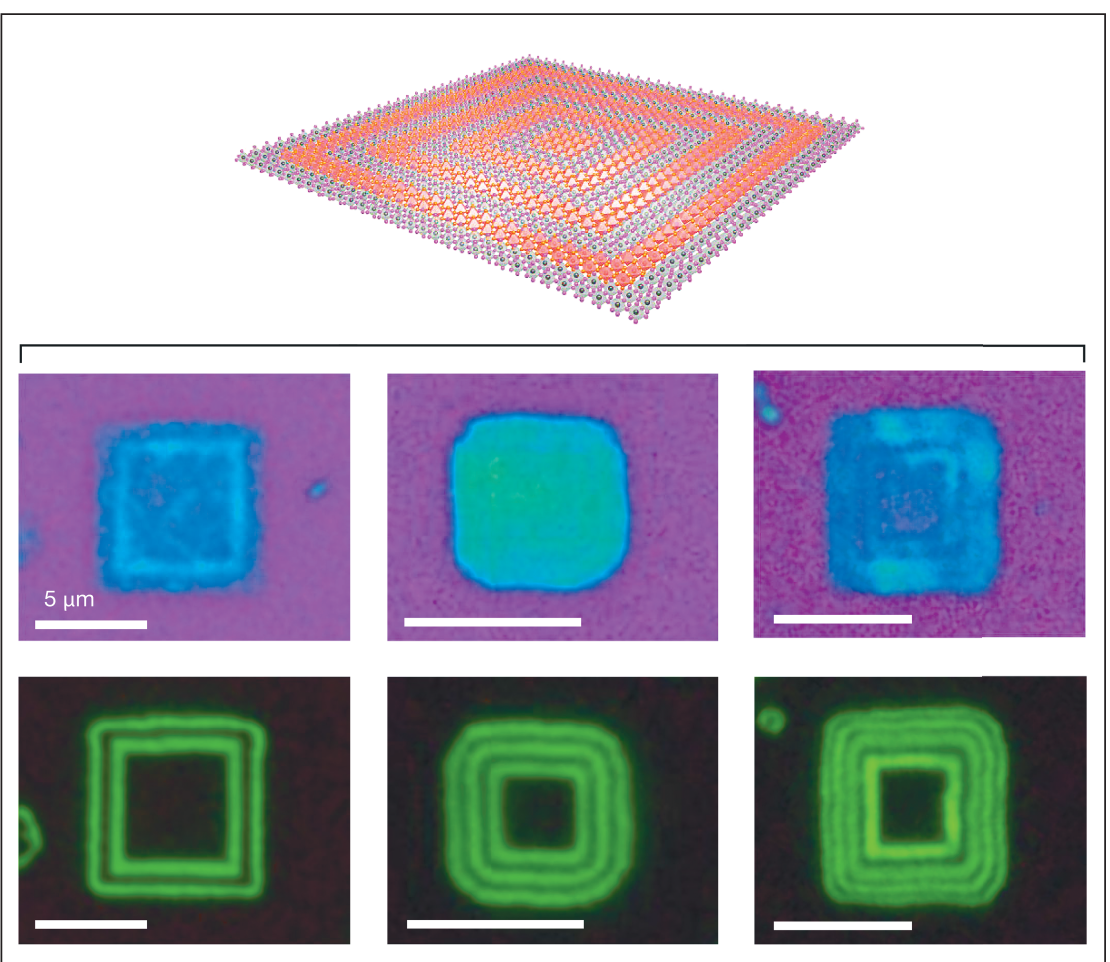

The top image is a schematic for a lateral superlattice of a 2D halide perovskite. The bottom panels have optical and photoluminescence images showing the formation of a lateral multi-heterostructure. Credit: Nature.

and epitaxial growth of halide perovskite films. The authors demonstrated highly tunable and stable lateral epitaxial halide perovskite heterostructures, multi-heterostructures, and superlattices.

The authors propose that the generic synthesis of $2 \mathrm{D}$ halide perovskite heterostructures presented in this work provides a strong platform to further advance the crystal chemistry of halide perovskites, exploration of optoelectronic properties, and eventually their applications in optoelectronic devices, such as diodes, lasers, and photovoltaics. 
$\mathrm{W}$ ide-bandgap perovskite and $\mathrm{Si}$ tandem solar cells have the ability to achieve $>30 \%$ power-conversion efficiency (PCE) at low cost. However, wide-bandgap perovskite materials, containing more than one type of halide ion, often have poor stability, owing to phase segregation under illumination. Solar cells with these wide-bandgap materials also show poor open-circuit voltage $\left(\mathrm{V}_{\mathrm{OC}}\right)$ efficiency.
$\mathrm{Xu}, \mathrm{McGehee}$, and co-workers, in their work published in Science (doi:10.1126/science.aaz5074), showed that the phase segregation in widebandgap halide perovskites can be suppressed by using triple-halide (chlorine, bromine, iodine) alloys even at 100-sun illumination intensity. This combination of triple halides also yields a 1.67 $\mathrm{eV}$ wide-bandgap material, which is a suitable match for tandem applications with crystalline Si solar cells. The authors achieved $>27 \%$ PCE in a two-terminal monolithic tandem solar cell with an area of $1 \mathrm{~cm}^{2}$.

The authors highlight that the triple halide-based perovskites, which show suppressed phase segregation even at high intensity illumination, can have applications beyond tandem solar cells, particularly in LEDs and concentrated photovoltaics.
$\mathrm{T}$ andem solar cells, where solar cells of different bandgaps are stacked to harness the different parts of the solar spectrum, present an opportunity to overcome the single-junction solar-cell limit. Solution-processed perovskite and c-Si solar cells together present an opportunity for large-scale tandem solar-cell fabrication. Industry-relevant crystalline silicon solar cells often have pyramidal textured surfaces to improve their photon harnessing capability. However, solution processing of a standard perovskite solar cell on top of a textured Si cell for monolithic integration is a difficult task, owing to the nonconformal coating of the thin perovskite layer.

De Wolf, Sargent, and co-workers have found a way to make perovskite and textured Si tandem cells with a certified efficiency of $25.7 \%$. This tandem cell showed a negligible loss in performance after a thermal stability test was completed for 400 hours at $85^{\circ} \mathrm{C}$.
The authors used micrometer-thick perovskite layers, which can have a detrimental effect on the charge collection efficiency. To overcome that issue, the authors enhanced the depletion width at the base of the Si pyramid texture. They used 1-butanethiol, a passivating agent, on the perovskite surface, which increased the carrier-diffusion length and suppressed phase segregation in the perovskite film. This work was published in Science (doi:10.1126/science.aaz3691).
Kim, Zhu, Kim, Shin, and coNworkers reported in Science (doi:10.1126/science.aba3433) a widebandgap perovskite and $\mathrm{Si}$ tandem solar cell with a PCE of $26.7 \%$. The perovskite material used in this tandem cell is $\left(\mathrm{FA}_{0.65} \mathrm{MA}_{0.2} \mathrm{Cs}_{0.15}\right) \mathrm{Pb}\left(\mathrm{I}_{0.8} \mathrm{Br}_{0.2}\right)_{3}$ with a $1.7 \mathrm{eV}$ bandgap. The singlejunction solar cell made with the widebandgap perovskite material retained
$80 \%$ of its initial PCE after 1000 hours of continuous illumination.

The authors used phenethylammonium (PEA)-based 2D additives to control the structural and electrical properties of the 2D passivation layer, which is based on a $\mathrm{PbI}_{2}$-framework. The PEAbased additives have two anion components, I and thiocyanate (SCN). The authors varied the anion composition and found that the I to SCN ratio of 1:3 was optimum. During the preparation of the perovskite thin films, the authors added an extra $2 \mathrm{~mol} \%$ of $\mathrm{Pb}(\mathrm{SCN})_{2}$ to the precursor solution to facilitate growth of the three-dimensional phase. The presence of the 2D perovskite phases at grain boundaries resulted in good electronic properties and a high solar-cell efficiency.

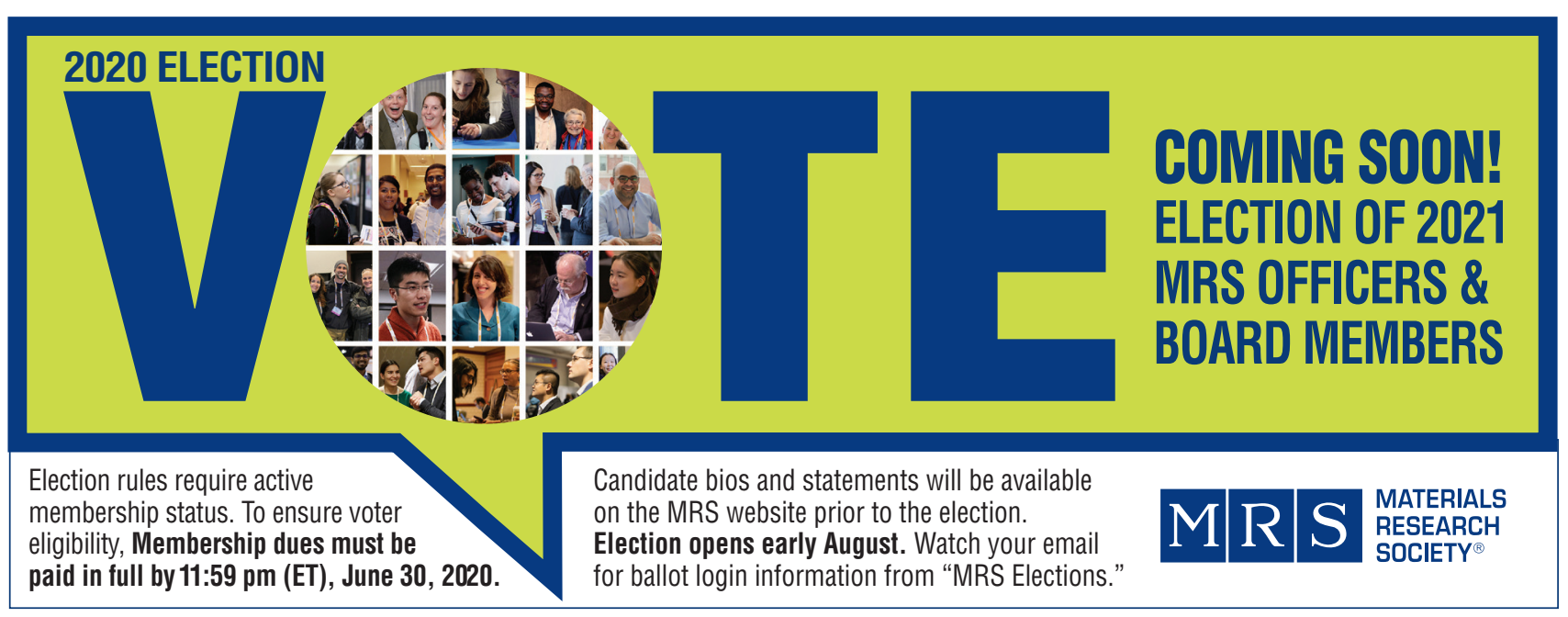

